Keywords: Cancer; Oncology; Depression; Previous trauma; Well-Being.

\title{
The association between previous psychological trauma and mental health among gastric cancer patients
}

\author{
Menachem Ben-Ezra* \\ Yuval Palgi* \\ Amit Shrira ${ }^{\star \star \star}$ \\ Yael Abrahami* \\ Etti Hodedi*

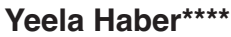 \\ Jonathan Jacob Wolf ${ }^{\star \star \star \star *}$

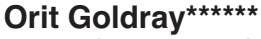 \\ Einat Shacham-Shmueli ${ }^{\star \star \star \star \star \star}$ \\ * School of Social Work, Ariel University \\ Center of Samaria, Ariel, Israel \\ ** Deparment of Gerontology, University of \\ Haifa, Haifa, Israel \\ *** Department of Psychology, Tel Aviv \\ University, Tel Aviv, Israel \\ **** Department of Psychology, Tel Aviv-Jaffa, \\ Academic College, Tel Aviv, Israel \\ ***** Department of Psychology, Trinity \\ College Dublin, Dublin, Ireland

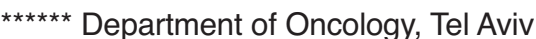 \\ Sourasky Medical Center, Sackler Faculty of \\ Medicine, Tel Aviv University, Tel Aviv, Israel \\ ISRAEL \\ IRELAND
}

\begin{abstract}
Background and Objectives: This study explores the association between previous trauma and psychiatric symptoms and well-being among gastric cancer patients. Based on the vulnerability model, it was hypothesized that cancer patients who report a history of trauma would exhibit higher level of psychiatric symptoms and lower well-being.

Methods: A sample of 123 consecutive cancer patients was interviewed and administered a battery of questionnaires in order to assess their psychiatric symptoms, well-being along with demographics and health behaviors in a cross-sectional design. The patients were divided based on previous exposure to psychological trauma. A set of hierarchical linear regressions was conduced in order to learn the association between previous trauma and mental health.

Results: Patients who reported previous exposure to trauma did not differ in the level of psychiatric symptoms from patients who did not report previous history of psychological
\end{abstract}


trauma. However, the former had significantly lower future life satisfaction and lower level of perceived social support. After controlling for covariates, patients who reported previous exposure also had higher levels of depressive symptoms.

Conclusions: The study results may suggest that the association between previous trauma and mental health of gastric cancer patients is more complex. Cancer patients who report a history of psychological trauma and who face an immediate threat to life do not differ on most indices, but they do show specific vulnerabilities: they are more susceptible to depressive symptoms and to lower future life satisfaction.

\section{Introduction}

In 2000, gastric cancer was the second most frequent cause of cancer death worldwide and the fourth most common cancer, with an estimated 650,000 deaths and 880,000 new cases per year ${ }^{1}$. Generally, the impact of being diagnosed with cancer leads to lower adjustment, quality of life and mental health ${ }^{2}$. The aftermath of being diagnosed with cancer has a drastic impact on the patient's life. This impact has both psychiatric and psychological aspects.

From a psychiatric point of view, cancer patients are at risk for mental disease and severe psychological distress due to their condition. Previous studies reported that mental health problems experienced by cancer patients most commonly involve depression and anxiety ${ }^{3,4}$. The incidence of adjustment disorder and depression has generally been reported to range from $15 \%$ to $40 \%$ in cancer patients ${ }^{5,6}$. However, an early review ${ }^{7}$ reported a wider range extending from $4.5 \%$ to $58 \%$, with an incidence rate still higher than in the general population one year after treatment. A longitudinal study ${ }^{8}$ found that across six points of measurement during one year, the rate of anxiety among esophagus or gastroesophageal junction cancer patients was $16 \%$ to $34 \%$ while for depression the scores were a little bit higher and ranged from $27 \%$ to $44 \%$. The main conclusion of the study was that psychiatric morbidity is common among esophageal cancer patients, both at inclusion and over time ${ }^{8,9}$. Summarizing reviews of depression in cancer raise a challenge as depressive symptoms occur on a broad spectrum that ranges from sadness to major affective disorder and because affect changes drastically making it difficult to assess when a patient is in a constant threat for life ${ }^{10}$. This review also found that among gastric cancer the rates of depression varies from $13 \%-50 \%$ which is similar to earlier reports ${ }^{10}$. Methodological issues such as lack of standardization in terms of population studied, type of cancer, disease stage, sample size, assessment instruments, cutoff score, type of interview, and diagnostic criteria employed (including post traumatic stress disorder, major depression on the clinical scale as to stress symptoms and depressive symptoms on a self rated assessment scales) all contributed to the large variance in reported prevalence among these studies $^{10-12}$. Additionally, the information about depression rates among gastric cancer is scarce in comparison to breast cancer ${ }^{10-12}$. 
Another important issue related to cancer diagnosis, is the history of trauma which serves as a risk factor for subsequent mental disorders, especially posttraumatic stress disorder (PTSD) and depression ${ }^{13}$. Previous trauma is a determinant for lower mental health among cancer patients in comparison to cancer patients without a history of trauma ${ }^{13-16}$. Moreover, a different study showed that a history of stressful or traumatic life events was associated with a shorter disease-free interval, indicating a potential harmful effect of these experiences on host resistance to tumor growth ${ }^{17}$. This may lead to an understanding that previous trauma may increase vulnerability for more negative outcomes when later stressors occur. From a psychological point of view, in many cases in which a cancer patient is being diagnosed, the psychological effect of immediate threat to life shatters the core beliefs and assumptions that the world is benevolent, just, controllable, and predictable ${ }^{18,19}$. This vulnerability is a risk factor for mental disorders, especially stress-related illness and depression. This can also lead to poorer mental health that can be viewed as a psychological marker of global vulnerability in terms of one's world assumptions and one's sense of optimism and hope ${ }^{18-21}$.

In sum, being diagnosed with cancer or being a cancer patient are considered vulnerable for PTSD symptoms and PTSD as well as for depressive symptoms and major depression ${ }^{10,11,22,23}$.

The purpose of our study was to examine the association between previous trauma and the mental health of gastric cancer patients. This association is understudied. Moreover, studies on psychiatric symptoms and psychological distress of gastric cancer patients are scarce. In a recent review of stress response syndrome and cancer, it was claimed that not only that this issue has re- cently began to receive research attention, but that very few of these studies refer to gastric cancer patients ${ }^{24}$. This is important therefore to explore this population in light of the high prevalence of gastric cancer and their high mortality rate ${ }^{1}$. Although gastric cancer is frequent, there is a lack of attention to the psychiatric sequelae of being a gastric cancer patient.

Based on the vulnerability model, we hypothesized that gastric cancer patients with a history of trauma would have a higher level of psychiatric symptoms (higher level of PTSD and depressive symptoms) and lower well-being (lower past, present, and future life satisfaction) than patients without a history of trauma.

\section{Method}

\section{Participants}

Participants were 123 consecutive gastric patients recruited from the Oncology unit at Tel Aviv Sourasky Medical Center while waiting for treatment. The patients were recruited during January-March 2008. Patients were included in the study if they met the following criteria: 1) age above 18 ; 2) no history of substance abuse or dependence; 3 ) not suffering from current cognitive impairment (receiving a score of less than 23 on the Mini-Mental State Examination). The mean age was $57.31(S D=12.74), 56.9 \%$ of the sample were men $(n=70)$, and $81.3 \%$ were married $(n=100)$. Beyond basic demographics, most of the patients $(77.2 \%, n=95)$ had Stage 3 cancer. Other patients had Stage 1 $(4.1 \%, n=5)$, Stage $2(13.8 \%, n=17)$, and Stage 4 cancer $(4.1 \%, n=6)$. The mean time since cancer diagnosis was 13.28 months $(S D=18.73)$. When asked whether they had any prior psychological trauma that was de- 
fined as experience of an event that led to an immediate threat to life (corresponding criterion A1 for PTSD in the DSM IV), $17.1 \%$ $(n=21)$ of the patients answered positively. The sample was divided into two groups based on prior traumatic experience. The demographics of the two groups are shown in Table 1. No significant differences were found between the groups in basic demographics (age, gender, and marital status). The study was approved by the Helsinki committee at Tel Aviv Sourasky Medical Center. All participants gave their informed consent.

\section{Measures}

The participants provided demographical data (age, gender, and marital status), and answered the following questions: "Have you experienced, witnessed, or was confronted with an event or events that involved actual or threatened death or serious injury, or a threat to the physical integrity of self or others?" (question based on DSM-IV, PTSD diagnosis, Criterion A1) coded as "no" (0) or "yes" (1), "Currently, do you feel that you receive sufficient social support from your close friends and relatives?" coded as "no" (0) or "yes" (1), and "Currently, what is your pain level on a scale of 0 to 10 where zero represents no pain and ten represents excruciating pain?" Participants also rated the following statements: "I adhere to the hospital personnel orders," rated "not at all" (1), "to some extent" (2), "much" (3), or "unconditionally" (4), "I take my medication on a regular basis" rated "not at all" (1), "to some extent" (2), "much" (3), or "unconditionally" (4). These questions are based on the modified simplified medication adherence questionnaire (SMAQ) ${ }^{25}$.

In addition, information regarding stage and time since cancer diagnosis (in month) was taken from each patient medical record.
During the interview, each participant filled a battery of commonly used questionnaires that assess PTSD symptoms, depressive symptoms, psychological distress and well-being.

PTSD symptoms were measured by the PTSD Checklist - Civilian Version (PCLC) [26], which uses 17 items addressing the DSM-IV $^{27}$ diagnostic criteria for PTSD. Participants use a five-point scale to evaluate each symptom in the last week. Higher scores indicate greater post-traumatic symptomatology. Cronbach's alpha coefficient for the PCLC in this sample was 0.92.

Depressive symptoms were measured by the Short Center for Epidemiologic Studies Depression Scale (SCESD) ${ }^{28,29}$ which is a 10 -item scale intended to measure the severity of depressive symptoms an individual experiences in the last week. The items are rated by frequency on a four-point scale, where higher scores reflect higher risk for depression. The Cronbach's alpha coefficient in this sample was 0.85 .

Psychological distress was measured by the General Health Questionnaire (GHQ) $)^{30}$. This scale contains 12 items for 12 specific psychological symptoms such as anhedonia and personal competence. Each item is rated by its frequency over the past few weeks on a four-point scale. Higher scores indicate poorer mental health. Cronbach's alpha coefficient for this sample was 0.87 .

Satisfaction with Life was measured by Cantril's Self Anchoring Scale (SAS) ${ }^{31}$. Participants were presented with a vertical ladder of 11 rungs, where the top (10) and the bottom ( 0 ) represented the best and worst possible conditions of one's life, respectively. The participants were asked to indicate on which rung they believed that they stood at the present time, has stood five years before, and would stand five years hence. 


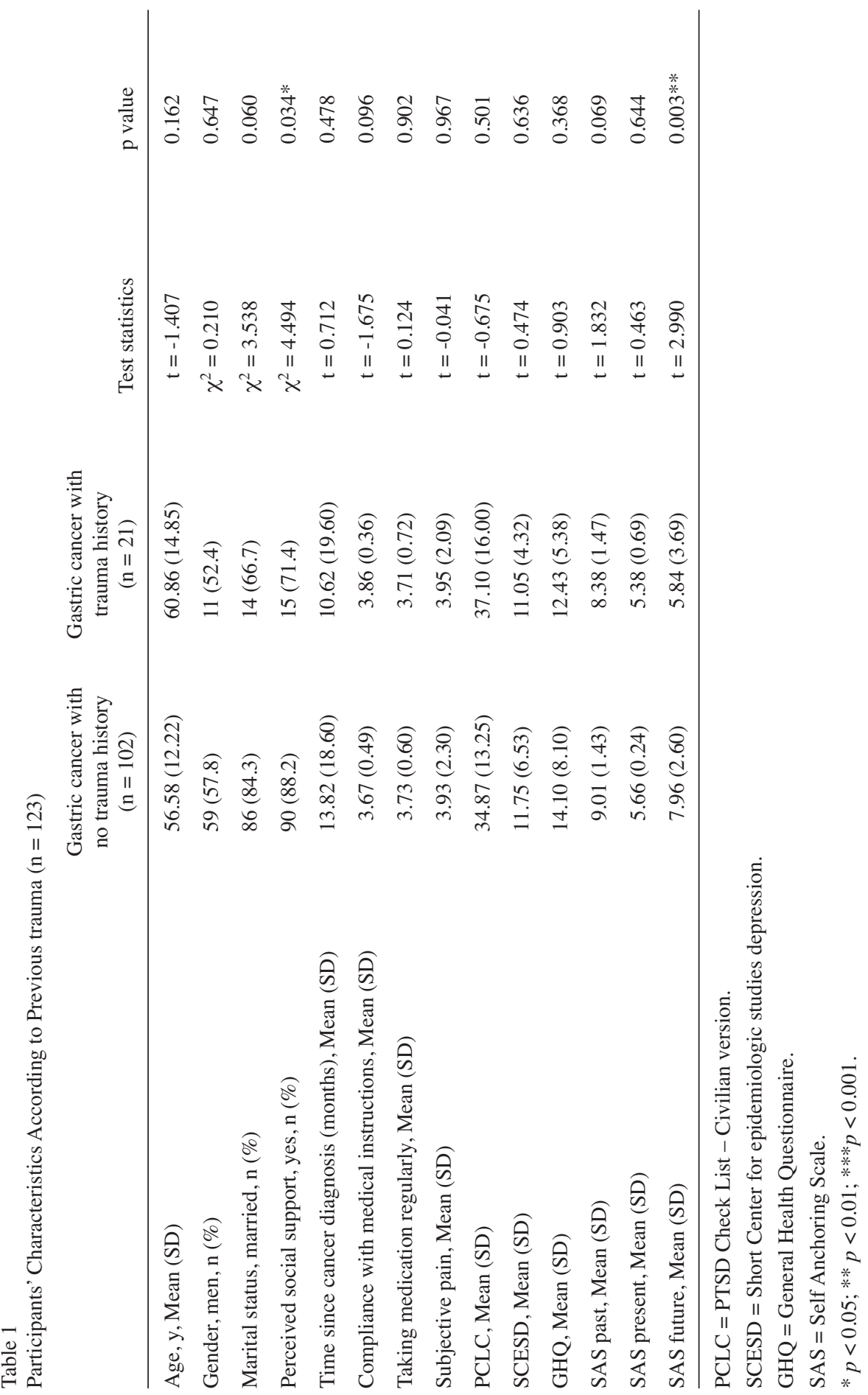




\section{Data Analysis}

The groups were compared on the study variables using $t$ and chi-square tests. Afterwards, six hierarchical linear regression analyses were conducted. Each hierarchical regression had the following logical steps: First step included the socio-demographic factors into the equation along with social support. The second step included cancer related factors in order to see the contribution of medical factors to the patients' mental health beyond basic demographics. The third step was the insertion of previous trauma. This factor was inserted last in order to see if there is a significant effect beyond basic demographic and cancer related factors.

In the first three regressions the dependent variables were PCLC, SCESD, and GHQ scores. The fourth regression was SAS past with three steps as presented earlier. The fifth regression used SAS present as dependent variable with four steps. The first step included demographics and perceived social support, the second step included health-related items, the third step included well-being (SAS past), and the fourth step included previous trauma. The sixth regression used SAS future as dependent variable with four steps. The first step included demographics and perceived social support, the second step was based on health-related items, the third step included well-being (SAS for present and past), and the fourth step included previous trauma.

All the analyses were conducted using SPSS program (SPSS, version 16, Chicago, IL).

\section{Results}

The results revealed no differences between the groups in basic demographics, time since cancer diagnosis, adhering to the hospital personnel orders, taking medications regularly, subjective pain, and mental health measurements, excluding perceived social support $\left(\chi^{2}=4.494 ; p=0.034\right)$, and SAS future scores $(t=2.990 ; p=0.003)$. Patients who did not report a previous trauma reported higher perception of social support and higher future life satisfaction relative to patients who reported a previous trauma. For more details, see Table 1.

Looking at the scores in a comparative way revealed that PTSD symptoms were higher in this study (34.87 and 37.10 respectively - measured by the PCLC) in comparison to another study on cancer patients (28.75 and 27.78 respectively - measured by the PCLC in two samples of cancer survivors $)^{32}$. The scores of depressive symptoms were similar in this study $(11.75$ and 11.05 respectively - measured by the SCESD) in comparison to another study on cancer patients measured in two time points (10.9 and 12.8 respectively - measured by the CES-D) and higher than those measured in a healthy comparison group (8.1 and 7.8 respectively - measured by the CES-D) $)^{33}$. The GHQ-12 scores (14.10 and 12.43 respectively) were similar to another study of cancer patients (13.09 in a combined sample of male and female cancer patients $)^{34}$. The scores of Cantril's self anchoring scale past were a little bit higher (9.01 and 8.38 respectively) in comparison to a study of breast cancer patients and comparison group (8.0 and 7.9 respectively) ${ }^{35}$. Additionally, the SAS present scores in our study were lower (5.66 and 5.38 respectively) in comparison to a study of breast cancer patients and comparison group (7.1 and 6.2 respectively $)^{35}$, and much lower on SAS future (7.96 and 5.84 respectively) in comparison to the same study (9.0 and 8.6 respectively $)^{35}$.

The first hierarchical regression revealed that PCLC scores were predicted by gender $(p<0.001)$, perceived social 
support $(p<0.01)$, and subjective pain $(p<0.01)$. The second hierarchical regression revealed that SCESD scores were predicted by gender $(p<0.05)$, marital status $(p<0.001)$ and reporting a previous trauma $(p<0.05)$. The third hierarchical regression revealed that GHQ scores were predicted bygender $(p<0.05)$, marital status $(p<0.01)$, and taking medications regularly $(p<0.05)$. For more details, see
Table 2. In sum, being a woman, having higher perceived social support and higher subjective pain were associated with higher PCLC scores. Being a woman, not married, and having a previous trauma were associated with higher SCESD scores. Being a woman, and not married were associated with higher GHQ scores, whereas, taking medications regularly was associated to lower GHQ scores.

Table 2

Results for the Hierarchical Regression Analysis Predicting PCLC, SCESD, and GHQ scores

\begin{tabular}{|c|c|c|c|}
\hline & PCLC & SCESD & GHQ \\
\hline Variable & $\beta$ & $\beta$ & $\beta$ \\
\hline \multicolumn{4}{|l|}{ Step 1} \\
\hline Age & 0.014 & 0.070 & 0.006 \\
\hline Gender & $0.312 * * *$ & $0.195^{*}$ & $0.214^{*}$ \\
\hline Marital status & 0.106 & $0.394 * * *$ & $0.292 * *$ \\
\hline Perceived social support & $0.249 * *$ & 0.115 & 0.068 \\
\hline$\Delta R^{2}$ & $0.212 * * *$ & $0.243 * * *$ & $0.157 * * *$ \\
\hline$R^{2}$ & $0.212 * * *$ & $0.243 * * *$ & $0.157 * * *$ \\
\hline \multicolumn{4}{|l|}{ Step 2} \\
\hline Time since cancer diagnosis & 0.062 & -0.012 & 0.008 \\
\hline Compliance with medical instructions & -0.076 & -0.051 & -0.132 \\
\hline Taking medication regularly & -0.016 & -0.057 & $-0.216^{*}$ \\
\hline Subjective pain & $0.275^{* *}$ & 0.058 & 0.040 \\
\hline$\Delta R^{2}$ & $0.069 *$ & 0.006 & 0.052 \\
\hline$R^{2}$ & $0.281 * * *$ & $0.249 * * *$ & $0.209 * * *$ \\
\hline \multicolumn{4}{|l|}{ Step 3} \\
\hline Previous trauma & -0.032 & $0.178 *$ & -0.145 \\
\hline$\Delta R^{2}$ & 0.001 & $0.028 *$ & 0.018 \\
\hline$R^{2}$ & $0.282 * * *$ & $0.277 * * *$ & $0.227 * * *$ \\
\hline$F$ & 4.762 & 4.197 & 3.560 \\
\hline$(d f)$ & $(1,109)$ & $(1,109)$ & $(1,109)$ \\
\hline
\end{tabular}

Only additional variables are shown in the results of additional steps that followed Step 1 .

PCLC $=$ PTSD Check List - Civilian version .

SCESD $=$ Short Center for epidemiologic studies depression.

GHQ $=$ General Health Questionnaire.

$* p<0.05 ; * * p<0.01 ; * * * p<0.001$. 
The fourth hierarchical regression revealed that SAS past scores were predicted by age $(p<0.01)$. The fifth hierarchical regression revealed that SAS present scores were predicted by age $(p<0.01)$, gender $(p$ $<0.01)$, marital status $(p<0.001)$, taking medications regularly $(p<0.001)$, subjec- tive pain $(p<0.05)$, and SAS past $(p<$ $0.001)$. The sixth hierarchical regression revealed that SAS future scores were predicted by age $(p<0.05)$, marital status $(p<$ $0.05)$, SAS past $(p<0.001)$, and reporting a previous trauma $(p<0.01)$. For more details, see Table 3 . In sum, being younger

Table 3

Results for the Hierarchical Regression Analysis Predicting SAS past, present, and future scores

\begin{tabular}{|c|c|c|c|}
\hline \multirow[b]{2}{*}{ Variable } & SAS past & SAS present & SAS future \\
\hline & $\beta$ & $\beta$ & $\beta$ \\
\hline \multicolumn{4}{|l|}{ Step 1} \\
\hline Age & $-0.272 * *$ & $-0.248 * *$ & $-0.202 *$ \\
\hline Gender & -0.174 & $-0.261 * *$ & -0.176 \\
\hline Marital status & 0.015 & $-0.391 * * *$ & $-0.208^{*}$ \\
\hline Perceived social support & -0.129 & 0.030 & 0.062 \\
\hline$\Delta R^{2}$ & $0.135^{* *}$ & $0.302 * * *$ & $0.122 * *$ \\
\hline$R^{2}$ & $0.135 * *$ & $0.302 * * *$ & $0.122^{* *}$ \\
\hline \multicolumn{4}{|l|}{ Step 2} \\
\hline Time since cancer diagnosis & 0.096 & -0.035 & 0.027 \\
\hline Compliance with medical instructions & 0.083 & 0.124 & -0.027 \\
\hline Taking medication regularly & -0.055 & $0.324 * * *$ & 0.197 \\
\hline Subjective pain & 0.086 & $-0.198 *$ & -0.078 \\
\hline$\Delta R^{2}$ & 0.025 & $0.086^{* *}$ & 0.082 \\
\hline$R^{2}$ & $0.161^{* *}$ & $0.388^{* * *}$ & $0.152 * *$ \\
\hline \multicolumn{4}{|l|}{ Step 3} \\
\hline SAS past & N/A & $0.405 * * *$ & $0.429 * * *$ \\
\hline SAS present & N/A & N/A & 0.061 \\
\hline Previous trauma ${ }^{a}$ & 0.117 & N/A & N/A \\
\hline$\Delta R^{2}$ & 0.012 & $0.138 * * *$ & $0.134 * * *$ \\
\hline$R^{2}$ & $0.173^{* *}$ & $0.526 * * *$ & $0.285^{* * *}$ \\
\hline \multicolumn{4}{|l|}{ Step 4} \\
\hline Previous trauma & N/A & 0.058 & $-0.270 * *$ \\
\hline$\Delta R^{2}$ & N/A & 0.003 & $0.063^{* *}$ \\
\hline$R^{2}$ & $0.173^{* *}$ & $0.529 * * *$ & $0.348^{* * *}$ \\
\hline$F$ & 4.464 & 12.011 & 4.619 \\
\hline$(d f)$ & $(1,109)$ & $(1,107)$ & $(1,95)$ \\
\hline
\end{tabular}

a - Previous trauma that applied only to SAS past.

Only additional variables are shown in the results of additional steps that followed Step 1 .

SAS $=$ Self Anchoring Scale.

$* p<0.05 ; * * p<0.01 ; * * * p<0.001$. 
was associated with higher SAS past scores. Being younger, men, married, taking medications regularly, having less subjective pain, and having higher SAS past scores were associated with higher SAS present scores. Being younger, married, having higher SAS past scores and reporting no history of previous trauma were associated with higher SAS future scores.

To sum, the research hypotheses were partially confirmed. Although gastric cancer participants who reported a previous trauma had significantly lower levels of social support, higher depressive symptoms, and lower future life satisfaction, they did not differ from their counterparts on all other indices.

\section{Discussion}

The results of this study showed that gastric patients who reported a previous trauma also reported higher depressive symptoms, lower perceived social support, and lower future life satisfaction relative to patients with no previous trauma. Gender and marital status were the most salient predictors of psychiatric symptoms, while age, marital status and past life satisfaction were the most robust predictors of well-being. There was no significant association between PTSD symptoms, general psychological distress and previous trauma. These results were in part contrary to our hypotheses and also contradicted several studies that showed that previous trauma is a risk factor for mental health ${ }^{13,14}$. One possible explanation is set within the dose-response model for traumatic stress ${ }^{36}$ which postulates that the level of the stressor yields a stress response as a function of exposure magnitude. As most of the sample in the current study was composed from Stage 3 cancer patients, it is likely that most of them were under extreme stress due to their advanced illness. These high levels of stress may have covered previous psychiatric differences between those who reported a history of psychological trauma from those who did not. Overall, these results do not reflect a comprehensive vulnerability but specific vulnerabilities of depressive symptoms, and low perceived social support. This more intricate model was also validated in other traumatized groups such as Holocaust survivors ${ }^{37}$. It is important to mention that although not significant differences were found in depressive symptoms between cancer patients who reported or did not report a previous trauma in the t-test analysis, the difference did arise in the hierarchical regression. One explanation to these results may lie in the fact that when controlling for demographics and cancer related factors, the difference between the groups in depressive symptoms becomes more salient.

Participants with prior traumatic experience had lower expectation of future life satisfaction. Future life satisfaction is a marker of well-being and possibly reflects a perception of prospective subjective life, mental health, and subjective perception of cancer condition among cancer patients. This cognitive domain can also be perceived as a buffer from current illness that helps to protect core beliefs. It will not inhibit the breach of core beliefs, but it can possibly prevent core assumptions from being shattered. Although psychological trauma does not always shatter core beliefs, it weakens them so when trauma recurs, they can collapse and in turn lead to foreshortened, pessimistic future perspective.

Moreover, perceived social support is known in the trauma literature as one with salubrious effects, especially after trauma ${ }^{13}$. Perceived social support is known to be associated with lower level of psychological 
distress and PTSD symptoms ${ }^{13}$. One explanation to our results is that the lack of adequate social networks or any other form of support group may increase prior vulnerability (previous trauma) and make the person more susceptible to the exacerbate effect of future trauma. We may add here some developmental perspective, and saying that as the participants were in the midlife and young old ages, deteriorated health, enhanced social isolation(a lot of references are available for this) and therefore social support has a major contribution to one's psychological condition.

The study has three limitations. First, it used a cross-sectional design instead of a longitudinal one. Therefore, it is hard to determine whether previous trauma increased depressive symptoms and pessimism or whether higher levels of depressive symptoms and pessimism made previous trauma more salient and available to report. However, as cancer patients were at an advanced stage of illness and the mean time since diagnosis was about one year, the prospective one-year survival is grim, expected to be less than $20 \%^{38,39}$. Second, we used self-report indices. A clinical interview could have helped in diagnosing psychiatric disorders and assessment of past trauma. Third, the relatively small group of patients who reported a prior exposure to trauma may lead to an inadequate power to find significant differences between the groups. Future studies should map global mental health, psychiatric symptoms, and well-being among larger groups of cancer patients exposed to a prior trauma, preferably in longitudinal designs. In this notion, it is important to emphasize that the study scores differed from other studies which examined PTSD symptoms (measured by PCLC) and well being (measured by Cantril's self anchoring scale for past, present and future). Gastric cancer pa- tients may have a unique framework of mental health in comparison to other cancer patients. Future studies should further examine gastric cancer patients vs. patients with other types of cancer in order to learn if these differences were sporadic or present a different pattern of mental health and wellbeing that is more pronounced in gastric cancer patients. Furthermore, it is recommended to expand our study to cancer patients in Stage one and two that have better survival prospects.

The main conclusion of the study is that gastric cancer patients who report a previous trauma are generally resilient and tend to show specific vulnerabilities (having more depressive symptoms, perceiving less social support, and reporting a lowered sense of future satisfaction). Gastric cancer patients should be inquired for previous traumatic events in their lives and interventions may focus on enhancing their sense of well-being, hope and optimism.

\section{References}

1. Stewart BW, Kleihaus P(Eds.). World Cancer Report. Lyon: IARC Press; 1993.

2. Omne-Pontén M, Holmberg L, Burns T, Adami HO, Bergström R. Determinants of the psycho-social outcome after operation for breast cancer: Results of a prospective comparative interview study following mastectomy and breast conservation. Eur J Cancer 1992; 28A: 1062-1067.

3. McDaniel JS, Musselman DL, Porter MR, Reed DA, Nemeroff CB. Depression in patients with cancer: Diagnosis, biology, and treatment. Arch Gen Psychiatry 1995; 52 : 89-99.

4. Spiegel D. Cancer and depression. Br J Psychiatry1996; 168: 109-116.

5. Derogatis LR, Morrow GR, Fetting J, Penman D, Piasetsky S, Schmale AM, et al. The prevalence of psychiatric disorders among cancer patients. JAMA 1983; 249: 751-757. 
6. Parle M, Jones B, Maguire P. Maladaptive coping and affective disorders among cancer patients. Psychol Med 1996: 26: 735-744.

7. Carroll BT, Kathol RG, Noyes R, Wald TG, Clamon $\mathrm{GH}$. Screening for depression and anxiety in cancer patients using the hospital anxiety and depression scale. Gen Hosp Psychiatry 1993; 15: 69-74.

8. Bergquist H, Ruth M, Hammerlid E. Psychiatric morbidity among patients with cancer of the esophagus or the gastro-esphageal junction: a prospective, longitudinal evaluation. Dis Esophagus 2007; 20: 523-529.

9. Hammerlid E, Ahlner-Elmqvist M, Bjordal K, Biörklund A, Evensen J, Boysen M, et al. A prospective multicentre study in Sweden and Norway of mental distress and psychiatric morbidity in head and neck cancer patients. $\mathrm{Br}$ J Cancer 1999; 80: 766-774.

10. Massie MJ. Prevalence of depression in patients with cancer. J Natl Cancer Inst Monogr 2004; 32: 57-71.

11. Sellick SM, Crooks DL. Depression and cancer: An appraisal of the literature for prevalence, detection, and practice guideline development for psychological interventions. Psychooncology 1999; 8: 315-333.

12. Bottomley A. Depression in cancer patients: A literature review. Eur J Cancer Care 1998; 7: 181-191.

13. Green BL, Krupnick JL, Rowland JH, Epstein SA, Stockton P, Spertus I, et al. Trauma history as a predictor of psychologic symptoms in women with breast cancer. J Clin Oncol 2000; 18: 1084-1093.

14. Hantman S, Solomon Z. Recurrent trauma: Holocaust survivors cope with aging and cancer. Soc Psychiatry Psychiatr Epidemiol 2007; 42: 396-402.

15. Matsuoka Y, Inagaki M, Sugawara Y, Imoto S, Akechi T, Uchitomi Y. Biomedical and psychosocial determinants of intrusive recollections in breast cancer survivors. Psychosomatics 2005; 46: 203-211.

16. Baider L, Goldzweig G, Ever-Hadani G, Peretz T. Psychological distress and coping in breast cancer patients and healthy women whose parents survived the Holocaust. Psychooncology 2006; 15: 635-646.

17. Palesh O, Butler LD, Koopman C, Giese-Davis J, Carlson R, Spiegel D. Stress history and breast cancer recurrence. J Psychosom Res 2007; 63: 233-239.

18. Janoff-Bulman R. Shattered assumptions: Towards a new psychology of trauma. New York: Free Press; 1992.

19. Lerner MJ, Miller DT. Just world research and the attribution process: Looking back and ahead. Psychol Bull 1978: 85: 1030-1051.
20. Aday LA. Health status of vulnerable populations. Annu Rev Public Health 1994; 15: 487-509.

21. Breslau N, Peterson EL, Schultz LR. A second look at prior trauma and the posttraumatic stress disorder effects of subsequent trauma. Arch Gen Psychiatry 2008; 65: 431-437.

22. Kangas M, Henry JL, Bryant RA. Posttraumatic stress disorder following cancer. A conceptual and empirical review. Clin Psychol Rev 2002; 22: 499-524.

23. Black EK, White CA. Fear of recurrence, sense of coherence, and posttraumatic stress disorder in haematological cancer survivors. Psychooncology 2006; 14: 510-515.

24. Gurevich M, Devins GM, Rodin GM. Stress response syndromes and cancer: conceptual and assessment issues. Psychosomatics 2002; 43: 259-281.

25. Knobel H, Alonso J, Casado JL, Collazos J, Gonzalez J, Ruiz I, et al. Validation of a simplified medication adherence questionnaire in a large cohort of HIV-infected patients: the GEEMA Study. AIDS 2002; 16: 605-613.

26. Ruggiero KJ, Del Ben K, Scotti JR, Rabalais AE. Psychometric properties of the PTSD Checklist-Civilian Version. J Trauma Stress 2003; 16: 495-502.

27. American Psychiatric Association. Diagnostic and statistical manual of mental disorders. Washington, DC: American Psychiatric Association Press; 1994.

28. Radloff LS. The CES-D scale: A self-report depression scale for research in the general population. Appl Psychol Meas 1977; 1: 385-401.

29. Andresen EM, Malmgren JA, Carter WB, Patrick DL. Screening for depression in well older adults: evaluation of a short form of the CES-D (Center for Epidemiologic Studies Depression Scale). Am J Prev Med 1994; 10: 77-84.

30. Goldberg DP, Gater R, Sartorius N, Piccinelli M, Gureje O, Rutter C. The validity of two versions of the GHQ in the WHO study of mental illness in general health care. Psychol Med 1997; 27: 191-197.

31. Cantril H. The pattern of human concerns. New Brunswick, NJ: Rutgers University Press; 1965.

32. DuHamel KN, Ostrof J, Ashman T, Winkel G, Mundy EA, Keane TM, et al. Construct validity of the posttraumatic stress disorder checklist in cancer survivors: analyses based on two samples. Psychol Assess 2004; 16: 255-266.

33. Hann D, Winter K, Jacobsen P. Measurement of depressive symptoms in cancer patients: evaluation of the Center for Epidemiological Studies Depression Scale (CES-D). J Psychosom Res 1999; 46: 437-443.

34. Atesci FC, Baltalarli B, Oguzhanoglu NK, Karadag F, Ozdel O, Karagoz N. Psychiatric morbidity among can- 
cer patients and awareness of illness. Support Care Cancer 2004; 12: 161-167.

35. Carpenter JS. Self-esteem and well being among women with breast cancer and women in an age-matched comparison group. J Psychosoc Oncol 1997; 15: 59-81.

36. March JS. What constitutes a stressor? The "Criterion A" issue. In: Davidson JRT, Foa EB (Eds.). Posttraumatic Stress Disorder, DSM-IV and Beyond. Washington, DC: American Psychiatric Association Press; 1993. p. 37-54.

37. Shmotkin D. Vulnerability and resilience intertwined: A review of research on Holocaust survivors. In: Jacoby R, Keinan G (Eds.). Between stress and hope: From a disease-centered to a health-centered perspective. Westport, CT: Praeger; 2003. p. 213-233.

38. Cenitagoya GF, Bergh CK, Klinger-Roitman J. A prospective study of gastric cancer. 'Real' 5-year survival rates and mortality rates in a country with high incidence. Dig Surg 1998; 15: 317-322.

39. Heise K, Bertran E, Andia ME, Ferreccio C. Incidence and survival of stomach cancer in a high-risk population of Chile. World J Gastroenterol 2009; 15: 1854-1862.

Author for correspondence:

Dr. Menachem Ben-Ezra,

School of Social Work

Ariel University Center of Samaria

Ariel 40700

ISRAEL

Tel.: +972-3-9066390

Email: menbe@ariel.ac.il 